\title{
Caracterización de las contusiones cutáneas e identificación de factores de riesgo durante el manejo presacrificio de cerdos comerciales ${ }^{\#}$
}

\author{
Characterisation of skin bruises and identification of risk factors during \\ pre-slaughter handling in commercial pigs
}

LJ Varón-Álvarez ${ }^{\mathrm{a}}$, MH Romero ${ }^{\mathrm{b}^{*}}$, JA Sánchez ${ }^{\mathrm{b}}$

${ }^{a}$ Estudiante Maestría en Ciencias Veterinarias, grupo de Investigación en Ciencias Veterinarias CIENVET, Facultad de Ciencias Agropecuarias, Universidad de Caldas, Manizales, Colombia.

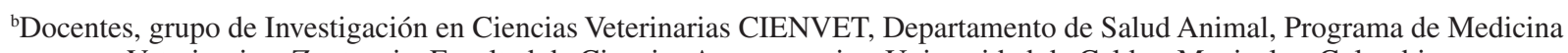
Veterinaria y Zootecnia, Facultad de Ciencias Agropecuarias, Universidad de Caldas, Manizales, Colombia.

\section{SUMMARY}

The goal of this study was to describe the gross characteristics of skin bruises and describe risk factors for pre-slaughter pigs. A total of 134 journeys $(3,156$ pigs) from a Colombian commercial slaughterhouse were surveyed. A multivariate logistic regression was used to assess the risk factors prior to slaughter. The condition of skin bruises was described as a binomial response (1: slight damage, 2: severe damage). The prevalence of skin bruises was $100.0 \%$, mean 6.1 bruises/carcass. Coma shaped bruises were most frequent (71.8\%), followed by linear (14.4\%), and diffuse shaped bruises (10.8\%). Factors such as mixing unfamiliar pigs in the farm, speed of the truck, prolonged journey stops, and lairage time $(\mathrm{P}<0.01)$ influenced the occurrence of skin bruises. Inspection of the animals during the journey decreased the prevalence of skin bruises.

Key words: animal welfare, meat quality, risk factor, slaughter.

\section{RESUMEN}

El objetivo del estudio fue describir las características generales de las contusiones cutáneas e identificar factores de riesgo durante el presacrificio porcino. Un total de 134 lotes (3.156 cerdos) fueron estudiados en una planta de sacrificio comercial colombiana. Se usó una regresión logística multivariada para identificar los factores de riesgo previos al sacrificio. Las contusiones cutáneas fueron descritas como una variable de respuesta binomial (1: daño leve, 2: daño severo). La prevalencia de contusiones fue $100 \%$, con un promedio de 6,1 contusiones/canal. Las contusiones cutáneas en forma de coma fueron las más frecuentes $(71,8)$, seguidas por las lineales $(14,4 \%$ y las difusas $(10,8 \%)$. La mezcla de animales no familiares en la granja, la velocidad del camión, las paradas prolongadas durante el transporte y el tiempo de estadía en la planta, estuvieron asociadas con la presencia de contusiones cutáneas $(\mathrm{P}<0,01)$. La inspección de los animales durante el viaje disminuyó la prevalencia de contusiones cutáneas.

Palabras clave: bienestar animal, calidad de la carne, factor de riesgo, sacrificio

\section{INTRODUCCIÓN}

Durante los últimos 25 años, el sector porcícola colombiano ha crecido hasta lograr importantes avances, que han permitido incrementar su participación en el mercado interno, aumentando el consumo de carne de cerdo por habitante en $31,4 \%$ durante el periodo 2000 - 2009, e incursionando en mercados internacionales (DNP 2007). La legislación Colombiana se encuentra en un proceso de modernización, en donde se ha integrado la cadena cárnica porcina con un enfoque "desde la granja,

Aceptado: 04.07.2013.

\# Financiado por la Vicerrectoría de Investigaciones y Posgrados de la Universidad de Caldas.

* Calle 65 No 26 - 10, Manizales, Caldas, Colombia; marlyn.romero@ucaldas.edu.co. hasta la mesa" (DNP 2007); se incluyeron estándares de calidad, inocuidad y bienestar animal (BA) en la producción primaria, el transporte de animales en píe (ICA 2007) y en el sacrificio (MPS $2007^{a, b}$ ).

Uno de los componentes más innovadores de la normatividad nacional son las prácticas de BA, las cuales se encuentran en un proceso incipiente de adopción en el presacrificio de animales de abasto público (Romero y col 2011 ). La Organización Mundial de Sanidad Animal (OIE) recomienda, dentro de sus directrices, evitar el uso de procedimientos violentos o medios que causen dolor o sufrimiento a los animales (OIE 2012). Las prácticas cruentas usadas durante el manejo de los cerdos tienen implicaciones éticas, económicas y sobre la calidad de la carne. Las canales provenientes de cerdos manejados inapropiadamente, presentan una mayor proporción de fracturas, hematomas, hemorragias petequiales y equi- 
móticas, que conllevan a un aumento en los decomisos durante la inspección sanitaria, disminución del valor comercial de la carne y mayor mano de obra para el procesamiento (Gispert y col 2000, Guárdia y col 2009). Por otra parte, estas prácticas son factores que favorecen la presencia de estrés agudo en los cerdos, inmediatamente antes del sacrificio, lo que causa un incremento en la tasa de acidificación post-mortem y por tanto, la presencia de carne pálida, flácida y exudativa (PSE), que genera grandes pérdidas a la industria porcina (Van de Perre 2010).

Durante el presacrificio los cerdos son expuestos a varias condiciones asociadas a manejo brusco, impacto violento contra estructuras agudas de camiones e instalaciones, ruptura de la estructura social y encuentros antagónicos entre los animales, así como altas densidades de carga durante el transporte, malas prácticas de conducción, condiciones geográficas adversas, entre otros factores, los cuales en conjunto favorecen la aparición de contusiones, producen estrés y afectan el bienestar animal (Guárdia y col 2009). Las contusiones en la piel han sido utilizadas como indicadores de BA en el presacrificio porcino (dalla Costa y col 2007, Correa y col 2010). El estudio de las características de las contusiones mediante el uso de protocolos estandarizados que registren la localización de la lesión en la canal, forma, severidad, número, color y tamaño, pueden ayudar a relacionar la causa y fuente de la contusión durante el presacrificio (Strappini y col 2009, Strappini y col 2012). La causa de la contusión puede estar relacionada con el grado de daño en la canal, siendo posible sugerir los eventos traumáticos a los cuales fueron sometidos los animales vivos e identificar los factores de riesgo que incrementaron su presentación. Por ejemplo, una canal con contusiones extensas y profundas en diferentes regiones anatómicas, sugieren que el animal fue pisoteado en el camión (Strappini y col 2012). La forma permite deducir con qué objeto se pudo haber ocasionado la contusión, es el caso de la contusión rectangular, en donde se observan lesiones individuales separadas, sugieren que la lesión fue producida por el uso de objetos contundentes como palos, durante el manejo y la conducción de los cerdos (dalla Costa y col 2007). En Colombia no existe ningún reporte de estudios que evalúen las condiciones de BA de cerdos sacrificados comercialmente y las características de las contusiones cutáneas, que permitan dilucidar los principales factores de riesgo del presacrificio y orienten las medidas de intervención requeridas para su fortalecimiento. El objetivo de la presente investigación consistió en caracterizar las contusiones cutáneas de cerdos comerciales e identificar factores de riesgo del presacrificio relacionados con su aparición.

\section{MATERIAL Y MÉTODOS}

\section{ANIMALES Y VARIABLES EVALUADAS}

Se realizó un estudio de corte transversal en donde fueron evaluados un total de 3.156 animales (134 viajes) en una planta de sacrificio comercial, localizada en la subregión norte del departamento de Antioquia (Colombia), durante los meses de julio y agosto de 2012. Mediante la aplicación de una encuesta estructurada dirigida a los transportadores $(\mathrm{n}=71)$ y la observación directa de los vehículos $(\mathrm{n}=134)$, se evaluaron las buenas prácticas de transporte exigidas por la legislación colombiana (ICA 2007). La descripción de las variables estudiadas se presenta en el cuadro 1. Los cerdos se movilizaron en camiones representativos para el transporte terrestre de ganado porcino en Colombia, vehículo de dos ejes con chasis rígido (combinado con madera y acero), provisto de ventilación pasiva, techo con carpa de lona y con una capacidad para 10 ton. Algunos vehículos estaban adaptados con dos pisos en su área de carga para el alojamiento de los porcinos. A la llegada de los cerdos a la planta de sacrificio fueron descargados por medio de una rampa móvil y alojados en corrales de recepción techados, protegidos de las condiciones climáticas cambiantes y provistos de suministro de agua fresca ad libitum, conservando el mismo grupo social proveniente de la granja. Los cerdos permanecieron toda la noche en la planta de sacrificio, fueron conducidos en lotes a través de un corredor de paredes compactas, insensibilizados en una cámara de $\mathrm{CO}_{2}$ automática, a continuación fueron izados y sangrados.

\section{MEDICIÓN Y CATEGORIZACIÓN DE CONTUSIONES}

Las contusiones cutáneas se caracterizaron en la línea de acabado del proceso, mediante la observación de las dos caras de la canal. Estas se clasificaron según la localización, el tamaño, la concentración, el color y la forma, de acuerdo con el protocolo propuesto por dalla Costa y col (2007), con modificaciones efectuadas por los autores. La clasificación fue realizada por un observador entrenado para evitar sesgos de medición. La localización de las contusiones en la canal se hizo teniendo en cuenta cinco regiones topográficas: cabeza, espalda, medio, lomo y jamón (figura 1). El tamaño de las contusiones cutáneas se clasificó en cinco categorías a saber: categoría 1 : entre $0,5-1 \mathrm{~cm}$, categoría 2: 2 - $5 \mathrm{~cm}$, categoría 3: 6 - $10 \mathrm{~cm}$, categoría 4: 11 - $15 \mathrm{~cm}$ y categoría 5: $>15 \mathrm{~cm}$. La variable concentración, tuvo en cuenta si las contusiones se encontraban agrupadas en un área específica de la canal o aisladas en partes separadas de la misma. La coloración se clasificó como roja, cuando las contusiones eran recientes, de aspecto fresco y color rojo; como marrón, cuando la coloración era oscura y estaban relacionadas con eventos antiguos. La forma se clasificó en cinco categorías: en coma, rectangular, lineal, difusa y como marca de la cámara de $\mathrm{CO}_{2}$, estas últimas caracterizadas por presentar forma romboide (figura 2).

\section{ANÁLISIS ESTADÍSTICO}

Se utilizó el número de contusiones cutáneas, como variable de respuesta binomial, la cual fue recodificada 
Cuadro 1. Descripción de variables evaluadas en el estudio.

Description of variables evaluated in the study.

\begin{tabular}{|c|c|}
\hline Variable & Descripción \\
\hline \multicolumn{2}{|l|}{ Del vehículo } \\
\hline$N^{\circ}$ de pisos & Uno o dos pisos \\
\hline Aristas & Presencia de bordes agudos en la carrocería \\
\hline Densidad de carga & $\mathrm{kg} / \mathrm{m}^{2}$ \\
\hline \multicolumn{2}{|l|}{ De las condiciones del viaje } \\
\hline Mezcla de animales en la granja & Lotes con animales de diferentes etapas \\
\hline Peso promedio & $\mathrm{kg}$ \\
\hline Velocidad promedio & Velocidad promedio $(\mathrm{km} / \mathrm{h})$ \\
\hline Paradas & Paradas prolongadas (más de $15 \mathrm{~min}$ ) del vehículo durante el transporte (Si o No) \\
\hline Hidratación & Durante el transporte (Si o No) \\
\hline Inspección de cerdos & Durante el transporte (Si o No) \\
\hline Tiempo de transporte & Duración total del recorrido finca-planta (h) \\
\hline Tipo de carretera & Pavimentado, Trocha, Mixto \\
\hline \multicolumn{2}{|l|}{ De la planta de sacrificio } \\
\hline Densidad en corral & $\mathrm{kg} / \mathrm{m}^{2}$ \\
\hline Disponibilidad en corral & Animales $/ \mathrm{m}^{2}$ \\
\hline Tiempo de estadía & Tiempo total desde la llegada a la planta hasta el sacrificio (h) \\
\hline \multicolumn{2}{|l|}{ Del animal } \\
\hline Tiempo de ayuno previo & Tiempo de ayuno en la finca $(\mathrm{h})$ \\
\hline Tiempo de ayuno total & Tiempo de ayuno en la fina + tiempo de ayuno (estadía) en planta (h) \\
\hline
\end{tabular}

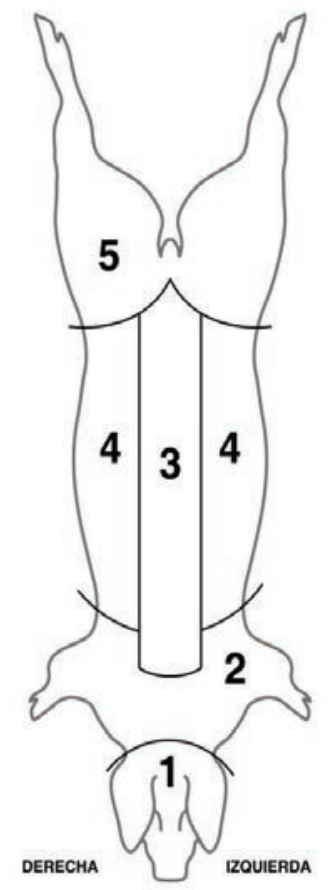

Figura 1. Esquema de evaluación de la canal. Los números indican las regiones anatómicas evaluadas. 1. Cabeza, 2. Espalda, 3. Lomo, 4. Medio, 5. Jamón.

Scheme of carcass assessment. Numbers indicate the anatomical regions evaluated. 1. Head, 2. Back, 3. Loin, 4. Middle 5. Ham. con valores de 1 (daño leve), que agrupaba las canales que presentaron entre 1 y 5 contusiones y 2 (daño severo), los rangos entre 6 y 13 contusiones. Los registros entre 9 y 13 contusiones se incluyeron en la categoría 2 (daño severo) por su baja frecuencia de presentación. Todos los valores de $\mathrm{P}<0,01$, fueron considerados como significativos. Se realizó una regresión logística, usando el programa STATA Versión 12,0 (College Station, Texas, EU). El modelo fue el siguiente:

$$
Y=\frac{e^{ß o+\sum ß i * ß X i}}{1+e^{ß o+\sum ß i * ß X i}}
$$

Donde $\mathrm{Y}=$ la probabilidad de contusiones cutáneas, $\beta 0$ el intercepto, $\beta 1$ los coeficientes de correlación y Xi el vector de las variables independientes incluidas en el análisis. Se realizaron análisis individuales para cada variable predictora, para explorar la información. A continuación, se ejecutó el modelo completo con las variables predictoras seleccionadas, para estimar sus efectos y grado de significancia. Las variables que no presentaron significancia fueron removidas una por una del modelo, comenzando por aquellas que presentaron un valor de $\mathrm{P}>$ 0,05 . Los modelos se volvieron a ejecutar para identificar las variables de confusión, por medio de la comparación de las estimaciones del nuevo modelo, con las estimacio- 


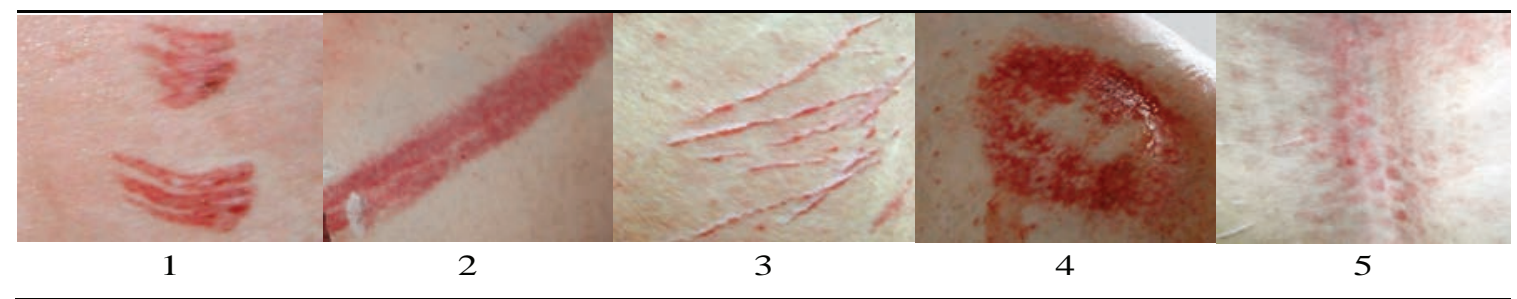

Figura 2. Clasificación según las formas de las contusiones evaluadas en la canal. 1. Coma, 2. Rectangular, 3. Lineal, 4. Difusa, 5. Romboide (cámara de CO2).

Classification according to shape of bruises evaluated in the carcasses. 1. Coma, 2. Rectangular, 3. Linear, 4. Diffuse, 5. Rhomboid (CO2 chamber).

nes del modelo anterior. Finalmente, se realizó la prueba de bondad de ajuste del modelo por medio del estadístico de Hosmer y Lemeshow. Los efectos de las variables predictoras sobre la aparición de contusiones cutáneas se expresaron por medio de las razones de dispariedad (OR) y sus respectivos intervalos de confianza del $95 \%$.

\section{RESULTADOS}

\section{CARACTERÍSTICAS GENERALES DEL TRANSPORTE Y ESTADÍA EN PLANTA}

Los camiones de dos pisos que transportan porcinos en Colombia no están dotados con rampas, pero la planta contaba con una rampa móvil en el área de descargue, que facilitaba esta labor. El 44,2\% de los cerdos fue transportado en camiones de un piso y el 55,8\% restante, en camiones de dos pisos, no se observaron diferencias significativas en la prevalencia de las contusiones de acuerdo a esta característica. Los vehículos en general cumplieron con los requisitos de la legislación sanitaria (ICA 2007). Los camiones estaban dotados de carpas para proteger a los animales de la condiciones climáticas adversas y presentaban pisos en materiales antideslizantes. El 30,8\% de los transportadores realizó paradas de larga duración (más de $15 \mathrm{~min}$ ), en tanto que el $34,2 \%$ se detuvo solamente el tiempo necesario para hacer la inspección de los animales durante el viaje. Sólo el 4,6\% de los lotes presentó mezcla de animales no familiares durante el transporte. Los cerdos fueron transportados en lotes de diferente tamaño: el 39,2\% conformado por uno a veinte animales, el 50,0\% entre 21 y 40 animales y el 10,8\% en grupos mayores de 40 cerdos (cuadro 2). La disponibilidad de espacio por animal exigida por la normatividad colombiana es de $1 \mathrm{~m}^{2}$ por animal en los corrales de recepción y sacrificio, aspecto que se cumplió en el presente estudio (MPS 2007 ${ }^{\mathrm{b}}$ ). El ayuno total promedio de los animales observados fue de 46,5 h; el 48,6\% de los cerdos tuvieron periodos de ayuno totales entre $48 \mathrm{~h}$ y $72,5 \mathrm{~h}$, mientras que para el 51,4\% restante el tiempo de ayuno total estuvo entre 24 y 48 h. El 25,1\% de los cerdos permaneció en la planta por un lapso superior a $24 \mathrm{~h}$ sin ser alimentados.

\section{PREVALENCIA Y DESCRIPCIÓN DE LAS CONTUSIONES}

El $100 \%$ de las canales presentó contusiones cutáneas, con un promedio de 6,1 contusiones/canal. La prevalencia de contusiones leves y severas fue 38,2\% ( $\mathrm{n}=$ $1.207)$ y $61,8 \%(n=1.949)$, respectivamente. Las contusiones más frecuentes estuvieron localizadas en el lomo $(32,0 \%)$ y el jamón $(22,0 \%)$. Predominaron las lesiones en forma de coma $(71,8 \%)$, lineal y difusa. En cuanto al tamaño, la concentración y el color, prevalecieron las contusiones con tamaños entre $2-10 \mathrm{~cm}(57,0 \%)$, agrupadas o concentradas en áreas específicas $(66,7 \%)$ y de color rojo (cuadro 3). Los cerdos que fueron transportados en camiones con dos pisos presentaron una mayor proporción de contusiones en sus canales $(55,2 \%)$, pero esta variable no fue considerada como un factor de riesgo $(\mathrm{OR}=1)$. La caracterización de las contusiones cutáneas se presenta en el cuadro 3. Por problemas del flameado, no fue posible evaluar el color de todas la contusiones localizadas en las cabeza.

\section{FACTORES DE RIESGO}

En el análisis de variables predictoras del modelo de regresión logística múltiple, la velocidad de transporte, la mezcla de cerdos no familiares en la granja, no inspeccionar los animales durante el viaje, realizar paradas prolongadas durante el transporte y la duración de la estadía en la planta, se consideraron influyentes sobre la presencia de contusiones cutáneas (cuadro 4). Los cerdos que fueron transportados en camiones a una velocidad superior a $50 \mathrm{~km} / \mathrm{h}$ tuvieron mayor probabilidad de presentar contusiones cutáneas, al compararse con los animales transportados a velocidades entre 20 y $40 \mathrm{~km} / \mathrm{h}$. No mezclar los lotes de cerdos no familiares en la granja antes del transporte, disminuyó el riesgo de presentar lesiones cutáneas $(\mathrm{OR}>1)$. Los porcinos que permanecieron en la planta por un tiempo mayor a $12 \mathrm{~h}$ presentaron mayor probabilidad de presentar contusiones cutáneas, que aquellos que estuvieron por menor tiempo, porque se observó que 
Cuadro 2. Media, desviación estándar (DE), mínimo y máximo para las variables continúas del estudio.

Mean, standard deviation (SD), minimum and maximum for continuous variables of the study.

\begin{tabular}{lcccc}
\hline \multicolumn{1}{c}{ Variable } & Media & DE & Mínimo & Máximo \\
\hline Densidad en el camión $\left(\mathrm{kg} / \mathrm{m}^{2}\right)$ & 217,4 & 72,9 & 45,4 & 428,8 \\
Disponibilidad de espacio en el camión (m²/animal) & 0,6 & 0,2 & 0,3 & 4,5 \\
Peso promedio $(\mathrm{kg})$ & 110,2 & 20,3 & 66,7 & 246,7 \\
Velocidad promedio $(\mathrm{km} / \mathrm{h})$ & 56,0 & 10,1 & 20,0 & 70,0 \\
Tiempo de transporte $(\mathrm{h})$ & 1,4 & 1,3 & 0,2 & 6,0 \\
Densidad en corral $\left(\mathrm{kg} / \mathrm{m}^{2}\right)$ & 93,6 & 35,3 & 8,6 & 181,7 \\
Disponibilidad de espacio en el corral (m²/animal) & 1,4 & 1,3 & 0,7 & 39,5 \\
Duración de la estadía en la planta de sacrificio $(\mathrm{h})$ & 18,8 & 7,7 & 4,6 & 38,8 \\
Tiempo de ayuno previo (h) & 24,3 & 10,4 & 8,0 & 48,0 \\
Tiempo de ayuno total $(\mathrm{h})$ & 46,5 & 11,7 & 18 & 72,5 \\
\hline
\end{tabular}

Cuadro 3. Características de las contusiones presentes en las canales evaluadas según el tipo de camión ( $\mathrm{N}=19.175$ contusiones). Characteristics of bruises present on carcasses evaluated according to the type of truck $(\mathrm{N}=19,175$ bruises $)$.

\begin{tabular}{|c|c|c|c|c|c|c|}
\hline \multirow{2}{*}{ Variable } & \multirow{2}{*}{$\mathrm{N}$} & \multirow{2}{*}{$\%$} & \multicolumn{2}{|c|}{ Un piso } & \multicolumn{2}{|c|}{ Dos pisos } \\
\hline & & & $\mathrm{n}$ & $\%$ & $\mathrm{n}$ & $\%$ \\
\hline \multicolumn{7}{|l|}{ Localización } \\
\hline Cabeza & 2.081 & 11,0 & 943 & 11,0 & 1.138 & 10,7 \\
\hline Espalda & 3.573 & 19,0 & 1.618 & 18,8 & 1.955 & 18,5 \\
\hline Lomo & 6.219 & 32,0 & 2.731 & 31,8 & 3.488 & 32,9 \\
\hline Medio & 3.085 & 16,0 & 1.428 & 16,6 & 1.657 & 15,6 \\
\hline Jamón & 4.217 & 22,0 & 1.864 & 21,7 & 2.353 & 22,2 \\
\hline \multicolumn{7}{|l|}{ Forma } \\
\hline Coma & 13.763 & 71,8 & 6.146 & 71,6 & 7.617 & 71,9 \\
\hline Rectangular & 30 & 0,2 & 21 & 0,2 & 9 & 0,1 \\
\hline Lineal & 2.063 & 10,8 & 889 & 10,4 & 1.174 & 11,1 \\
\hline Difusa & 2.752 & 14,4 & 1.264 & 14,7 & 1.488 & $1.4,0$ \\
\hline Marca de la Cámara de $\mathrm{CO}_{2}$ & 567 & 3,0 & 264 & 3,1 & 303 & 2,9 \\
\hline \multicolumn{7}{|l|}{ Tamaño $(\mathrm{cm})$} \\
\hline $0,5-1$ & 435 & 2,3 & 209 & 2,4 & 226 & 2,1 \\
\hline $2-5$ & 6.817 & 35,6 & 3.010 & 35,1 & 3.807 & 35,9 \\
\hline $6-10$ & 4.095 & 21,4 & 1.895 & 22,1 & 2.200 & 20,8 \\
\hline $11-15$ & 2.460 & 12,8 & 1.118 & 13,0 & 1.342 & 12,7 \\
\hline $15<$ & 5.368 & 28,0 & 2.352 & 27,4 & 3.016 & 28,5 \\
\hline \multicolumn{7}{|l|}{ Concentración } \\
\hline Juntas & 12.792 & 66,7 & 5.777 & 67,3 & 7.015 & 36,6 \\
\hline Aisladas & 6.383 & 33,3 & 2.807 & 32,7 & 3.576 & 18,6 \\
\hline \multicolumn{7}{|l|}{ Color ${ }^{a}$} \\
\hline Rojo & 17.158 & 97,2 & 7.690 & 96,9 & 9.468 & 97,5 \\
\hline Marrón & 488 & 2,8 & 245 & 3,1 & 243 & 2,5 \\
\hline
\end{tabular}

${ }^{a}$ Se evaluó el color de la contusión en 3.156 canales ( $\mathrm{n}=17.646$ contusiones). 
Cuadro 4. Coeficientes, error estándar (EE), intervalo de confianza (IC) y probabilidad (P) de las variables incluidas en el modelo de regresión logístico multivariado.

Coefficients, standard error (SE), confidence interval (CI) and probability $(\mathrm{P})$ of the variables included in the multivariate logistic regression model.

\begin{tabular}{|c|c|c|c|c|c|}
\hline Variable & Categoría & $\mathrm{OR}^{a}$ & $\mathrm{EE}$ & IC $95 \%$ & Valor $\mathrm{p}$ \\
\hline \multirow[t]{3}{*}{ Velocidad de transporte $(\mathrm{km} / \mathrm{h})$} & $20-40$ & 1,0 & & Ref & Ref \\
\hline & $50-60$ & 1,5 & 0,2 & $1,1-1,9$ & 0,02 \\
\hline & $70-80$ & 1,7 & 0,3 & $1,2-2,3$ & 0,02 \\
\hline \multirow[t]{2}{*}{ Mezcla de cerdos no familiares } & $\mathrm{Si}$ & 1,0 & & Ref & Ref \\
\hline & No & 0,6 & 0,1 & $0,4-0,8$ & $<0,01$ \\
\hline \multirow[t]{2}{*}{ Inspeccionar animales durante el viaje } & $\mathrm{Si}$ & 1,0 & & Ref & Ref \\
\hline & No & 1,4 & 0,2 & $1-1,9$ & 0,045 \\
\hline \multirow[t]{2}{*}{ Paradas prolongadas durante el transporte } & $\mathrm{Si}$ & 1,0 & & Ref & Ref \\
\hline & No & 0,6 & 0,1 & $0,4-0,8$ & 0,02 \\
\hline \multirow[t]{5}{*}{ Duración de la estadía en la planta (h) } & $<8$ & 1,0 & & Ref & Ref \\
\hline & $8,1-12$ & 0,7 & 0,1 & $0,5-1,0$ & 0,04 \\
\hline & $12,1-18$ & 1,2 & 0,2 & $0,8-1,5$ & NS \\
\hline & $18,1-24$ & 1,3 & 0,2 & $0,9-1,7$ & NS \\
\hline & $>24$ & 1,5 & 0,2 & $1,1-2$ & $<0,01$ \\
\hline
\end{tabular}

${ }^{a}(\mathrm{OR}=$ Razones de dispariedad; Ref: categoría considerada como referencia; NS: no significativo).

a medida que aumentó la duración de la estadía de los cerdos en la planta, se incrementó el riesgo de contusiones en las canales $(\mathrm{OR}>1)$ (cuadro 4).

\section{DISCUSIÓN}

Antioquia es considerada la región porcícola más importante de Colombia, porque concentra la mayor proporción de las explotaciones tecnificadas $(35,51 \%)$, es el departamento con el $46 \%$ de la producción nacional de carne de cerdo y cuenta con el consumo más alto de carne, representado en $13,79 \mathrm{~kg} /$ per cápita/año (DNP 2007). Este estudio constituye el primer reporte que evalúa indicadores de $\mathrm{BA}$ durante el presacrificio de cerdos en Colombia, cuyos resultados podrán ser aplicados a la cadena logística porcina. La presencia de contusiones cutáneas en las canales, ha sido relacionada con factores de riesgo durante el presacrificio porcino y se ha sugerido que las altas densidades de carga del camión y de los corrales durante la estadía en la planta de sacrificio, así como la duración de los mismos, incrementan el riesgo de presentación, aspectos que han sido evaluados en varios estudios desarrollados en Brasil, Italia y España (Nanni Costa y col 2002, Mota-Rojas y col 2006).

\section{CARACTERÍSTICAS GENERALES DEL TRANSPORTE Y ESTADÍA EN PLANTA}

El transporte es una de las etapas críticas para el BA y la calidad de la carne (Fitzgerald y col 2009). Estudios realizados en Colombia han evidenciado que el transporte de ganado no es una actividad especializada y los transportadores utilizan métodos cruentos, como palos, varillas de hierro y las varetas del camión para manejar los animales, aspecto que incrementa el riesgo de contusiones (Romero y col 2012). El ganado porcino en Colombia es transportado en camiones con carrocerías de madera simples (un piso) o dobles (dos pisos). A pesar de que el tipo de camión utilizado para transportar los cerdos ha sido considerado como un factor de riesgo para la presencia de contusiones y de mortalidad durante el transporte (dalla Costa y col 2007, Fitzgerald y col 2009), la prevalencia de contusiones cutáneas en la investigación, no presentó diferencias significativas de acuerdo con esa característica. De otra parte, en el estudio, las carrocerías se encontraban en buen estado y una pequeña proporción presentó aristas, lo cual se considera un aspecto positivo, que no estuvo asociado con la presencia de contusiones cutáneas. 
Se observó que las densidades de carga en los camiones no estaban estandarizadas y que se transportan animales tanto en altas como bajas densidades, lo cual sugiere que no existe una planificación del viaje, así como una coordinación entre los proveedores de los cerdos y la planta de sacrificio (Romero y col 2011 ${ }^{\text {b }}$, OIE 2012). La legislación colombiana reglamenta las necesidades mínimas de espacio para el transporte de cerdos, que oscilan entre $0,5 \mathrm{~m}^{2} /$ animal para cerdos de $100 \mathrm{~kg}$ de peso y de $0,8 \mathrm{~m}^{2} /$ animal, para animales adultos (ICA 2007). En el $37,3 \%$ de los casos la disponibilidad de espacio en los camiones no estuvo acorde con las exigencias oficiales (cuadro 2). Densidades muy altas generan gastos de energía adicionales, que se traducen en alteraciones del $\mathrm{pH}$ en la carne; los cerdos transportados a densidades muy bajas, cuentan con mayor disponibilidad de espacio, lo que favorece las caídas y las colisiones de los cerdos, contra la carrocería del camión y entre los coespecíficos. Esta variable no estuvo relacionada con la presencia de contusiones cutáneas en el estudio, como sí se reportó en el país durante el transporte de bovinos (Romero y col 2012) y en otros estudios en Norte América, donde la densidad de carga se ha asociado a pérdidas por lesiones (Fitzgerald y col 2009). Sin embargo, esta falta de asociación fue concordante con otros estudios en porcinos, donde se reporta que densidades de $0,4 \mathrm{~m}^{2} / 100 \mathrm{~kg}$, muy similares a las observadas en Colombia, tienen un efecto muy bajo sobre las contusiones cutáneas (Guárdia y col 2004).

Se ha observado que las contusiones cutáneas de los cerdos se incrementan en la medida que aumenta el tiempo de transporte y pueden llegar a duplicarse durante las últimas 6 a 8 h de viaje (Fitzgerald y col 2009, Pérez y col 2002). En el estudio, los tiempos de transporte tuvieron una duración promedio de 1,4h; las granjas eran parte de una cooperativa de productores, se encontraban localizadas en el área de influencia de la planta de sacrificio y los camiones utilizados para el transporte presentaron buenas condiciones de mantenimiento, aspectos que en conjunto pudieron incidir en la no asociación entre el tiempo de transporte y la presentación de contusiones en las canales. Así mismo, estuvieron acorde con los criterios exigidos por la legislación sanitaria, que establece que la duración del transporte de los cerdos, no debe ser mayor a $8 \mathrm{~h}$ (ICA 2007).

El periodo de ayuno de los cerdos previo al transporte hacia las plantas de sacrificio, no está reglamentado en Colombia, por lo que los productores seleccionan sus propios criterios (ICA 2007). En el estudio, este periodo osciló entre 8 y 48 h (cuadro 2), período que permite disminuir costos de alimentación para el productor, reduce la mortalidad de los cerdos durante el transporte, evita la contaminación de las canales durante el eviscerado y facilita la sangría (Warriss 2003). Los animales deben permanecer en la planta de sacrificio mínimo $4 \mathrm{~h}$, periodo que no puede exceder las $48 \mathrm{~h}$. Así mismo, la reglamentación exige el suministro de alimento a los cerdos, cuando el tiempo de estadía supera las 24 h (MPS 2007b). Este último aspecto no se cumplió en su totalidad en la planta evaluada, lo que sugiere que la privación prolongada de alimento y el estrés generado por la sensación de hambre, pudo inducir a encuentros antagónicos entre los animales y por tanto un incremento de las contusiones cutáneas (Rosenvold y Andersen 2003).

\section{PREVALENCIA Y DESCRIPCIÓN DE LAS CONTUSIONES}

La categorización de las contusiones permitió establecer que la mayor proporción de éstas fueron consideradas como severas y se localizaron principalmente en el jamón y el lomo, que son las áreas más expuestas del cerdo, en donde se ubican las lesiones que son infringidas por los operarios durante la movilización, lo que evidencia problemas de la relación hombre-animal y de BA, que producen sufrimiento en este proceso (Correa y col 2010). Las canales presentaron un promedio alto de contusiones cutáneas (6,1 contusiones/canal), comparadas con las halladas en canales provenientes de plantas de sacrificio con tiempos de estadía similares, que han implementado métodos humanitarios de manejo de los cerdos (2,9 contusiones/canal) (Zhen y col 2012).

Pese a que en la planta no se realizó la mezcla de los lotes de los animales al ingreso, ni durante la estadía en los corrales de recepción, fueron frecuentes las contusiones múltiples en forma de coma, concentradas en diferentes áreas y con tamaños entre 2 y $10 \mathrm{~cm}$, características de las producidas por mordeduras durante los encuentros antagónicos de los cerdos (figura 2). Esto pudo deberse a que la mezcla de animales no familiares se realiza durante el cargue en las granjas, práctica común en el área de estudio, por la heterogeneidad que algunas veces presentan los lotes en la fase final de la ceba, por lo cual los productores escogen los cerdos de pesos similares en cada lote (triaje) justo antes de ser llevados a la planta de sacrificio. Esta reagrupación social propicia la agresividad y el establecimiento de nuevas jerarquías en los corrales de las plantas de sacrificio, lo cual explica la elevada proporción de contusiones con las características anteriormente descritas (Rosenvold y Andersen 2003, Turner y col 2006). Se considera que las contusiones cutáneas en forma de coma localizadas en el jamón y en los hombros (regiones anatómicas 2 y 5 , figura 1), con tamaños entre 0,5 y $1 \mathrm{~cm}$, o largas y delgadas, entre 10 a $15 \mathrm{~cm}$, son causadas por las pezuñas de los cerdos durante las montas a sus coespecíficos y se relacionan con el hacinamiento (Correa y col 2010). Asimismo, la pequeña proporción de lesiones rectangulares encontrada pudieron estar relacionadas con la utilización de objetos contundentes durante el manejo. Aunque la presencia de contusiones en forma de rombo, producidas por el piso de la cámara de $\mathrm{CO}_{2}$, tuvo una baja prevalencia, éstas advierten problemas técnicos del equipo o una excesiva presión de la pinza de sujeción de los cerdos durante la insensibilización (figura 2). 
Predominaron las contusiones recientes o rojas $(97,2 \%)$, que indican que la mayoría de éstas se infringieron en la planta, durante las $10 \mathrm{~h}$ previas al sacrificio, es decir, durante la estadía en los corrales y la conducción hacia el área de insensibilización (Strappini y col 2009). Las lesiones se tornan marrones o rojas oscuras hacia las $24 \mathrm{~h}$ de haber sido provocadas, debido a los procesos inflamatorios que se llevan a cabo durante este periodo, en donde los macrófagos han ingerido las células sanguíneas y metabolizado la hemoglobina (Strappini y col 2009). No se evidenciaron contusiones de color amarillo, consideradas como lesiones provocadas días, o inclusive, semanas antes, cuya tonalidad se debe a la presencia de niveles de bilirrubina específicos (Strappini y col 2009).

\section{FACTORES DE RIESGO}

En los manejos previos al sacrificio los animales se enfrentan a numerosos factores estresantes en un periodo de tiempo relativamente corto; un nivel elevado de estrés desencadena efectos adversos tales como agresión, heridas y muerte (Van de Perre 2010). Con relación a las condiciones de transporte, la velocidad del camión fue considerada un factor de riesgo para la aparición de las contusiones cutáneas. Se encontró que a medida que la velocidad aumentaba, superando los $40 \mathrm{~km} / \mathrm{h}$, se incrementó la probabilidad de aparición de contusiones cutáneas. Los movimientos bruscos se relacionaron con la topografía montañosa del área de estudio, produciendo caídas y colisiones, por lo que se recomienda conducir a velocidades no superiores de los $60 \mathrm{Km} / \mathrm{h}$ (Romero y col 2011 a), porque las aceleraciones altas aumentan la frecuencia cardiaca de los cerdos y produce vibraciones de baja frecuencia, condiciones poco confortables para los animales (Berrecil-Herrera y col 2009). Debido a la presencia de carreteras de tipo mixto (asfaltadas y caminos rurales) en el área estudiada y a la costumbre de los conductores de no mantener una velocidad constante durante el viaje, sería conveniente que durante los primeros 15 a 20 min de viaje, los conductores conduzcan a bajas velocidades, para que los animales se habitúen a los movimientos del vehículo (Romero y col 2011 b).

Con relación al reagrupamiento social, no mezclar animales de diferentes camadas y/o etapas productivas en la granja antes del transporte, se identificó como un factor protector en el estudio, porque evita los encuentros antagónicos entre los cerdos y disminuye la probabilidad de encontrar contusiones cutáneas en las canales (cuadro 4). Con frecuencia durante el viaje, los transportadores hacen paradas prolongadas para ingerir alimentos y descansar. Esta variable aumentó la presencia de contusiones cutáneas en las canales evaluadas, lo cual pudo estar relacionado con un aumento de actividad y de encuentros antagónicos, debido al estrés del ambiente nuevo, aumento de la temperatura interna del vehículo y las condiciones propias del camión, entre otros aspectos (Nanni
Costa y col 2002). La Organización Mundial de Salud Animal (OIE) recomienda la revisión de los animales durante el transporte, lineamiento que es de obligatorio cumplimiento en Colombia (ICA 2007, OIE 2012). El objetivo de esta práctica consiste en identificar oportunamente animales convalecientes, agitados o caídos durante el viaje. En el estudio, se evidenció que no inspeccionar regularmente los animales incrementó el riesgo de presentar contusiones cutáneas $(\mathrm{OR}=1,4)$.

La estadía en la planta es una etapa decisiva en el BA, cuyo impacto depende tanto de la duración y de las condiciones de la estabulación, como la disponibilidad de espacio, el manejo, las condiciones propias de las instalaciones y el medio ambiente (Warriss 2003). La planta de sacrificio estudiada contaba con infraestructura y diseño moderno. Los corrales de recepción y de sacrificio de los cerdos estaban techados, provistos de pisos antideslizantes en cemento y con capacidad para albergar lotes completos, por tanto, no se presentó la mezcla de animales no familiares en esta fase. Así mismo, la duración de la estadía, la disponibilidad de espacio en los corrales y el suministro de agua ad libitum, estuvieron acordes con la legislación sanitaria (MPS 2007b). No obstante, se apreció que los tiempos de estadía en planta superiores a las $12 \mathrm{~h}$ incrementaron el riesgo de contusiones cutáneas, resultados descritos de igual forma por otros autores (Nanni y col 2002, Zhen y col 2012). Sin embargo, ha sido evidente que duraciones de estadía en planta, inferiores a las observadas en estos estudios, disminuyen la probabilidad de encontrar contusiones cutáneas en las canales (Guárdia y col 2004). Otro aspecto que sería importante evaluar en posteriores trabajos, es el tamaño del lote. En el estudio predominaron los lotes pequeños (entre 21 y 40 cerdos/corral); se ha descrito que los animales que pertenecen a grupos grandes, tienen mayor oportunidad de escapar de comportamientos agresivos, comparados con aquellos que se encuentran en grupos pequeños (Warriss 2003, Zhen y col 2012).

Los resultados del estudio permiten concluir que la evaluación de las contusiones cutáneas en canales porcinas pueden ser útiles indicadores de la pérdida de BA durante el presacrificio, relacionadas con problemas de manejo y de la interacción hombre-animal, principalmente. La caracterización de las contusiones permitió identificar problemas de manejo en el área de estudio, como la mezcla de animales de diferente origen en la granja, el estilo de la conducción (velocidad del camión), no inspeccionar los animales y efectuar paradas prolongadas (de más de $15 \mathrm{~min}$ ) durante el viaje, así como la duración de la estadía en la planta, considerados como factores de riesgo. Los cerdos evaluados fueron sometidos a largos tiempos de ayuno. Así mismo, fue evidente una relación negativa entre los manejadores y los cerdos, por la alta proporción de contusiones cutáneas de grado severo. Es necesario fortalecer la investigación del BA durante el presacrificio porcino, la capacitación y entrenamiento del 
personal responsable del manejo de los cerdos, divulgar la normatividad sanitaria vigente e integrar el BA como un componente esencial de la cadena cárnica porcina, orientada a obtener carne de mejor calidad organoléptica y ética, e inocua para el consumidor.

\section{AGRADECIMIENTOS}

Los autores agradecen a la Vicerrectoría de Investigaciones de la Universidad de Caldas por la financiación de la investigación y a Frigocolanta, por el soporte técnico y operativo.

\section{REFERENCIAS}

Becerril-Herrera M, D Mota-Rojas, I Guerrero, A Schunemann de Aluja, C Lemus-Flores, M González-Lozano, R Ramírez-Necoechea, M Alonso-Spilsbury. 2009. Relevant aspects of swine welfare in transit. Vet Méx 40, 315-329.

Correa JA, S Torrey, N Devillers, JP Laforest, HW Gonyou, L Faucitano. 2010. Effects of different moving devices at loading on stress response and meat quality in pigs. $J$ Anim Sci 88, 4086-4093.

Dalla Costa OA, L Faucitano, A Coldebell, JV Ludke, JV Peloso, D Roza, MJR Paranhos da Costa. 2007. Effects of the season of the year, truck type and location on truck on skin bruises and meat quality in pigs. Livest Sci 107 , 29-36.

Departamento Nacional de Planeación DNP, Consejo Nacional de Política Económica y Social, Colombia. 2007. Conpes 3458, Política nacional de Sanidad e Inocuidad para la cadena porcícola. Bogotá, Colombia.

Fitzgerald RF, KJ Stalder, JO Matthews, CM Schultz-Kaster, AK Johnson. 2009. Factors associated with fatigued, injured, and dead pig frequency during transport and lairage at a commercial abattoir. J Anim Sci 87, 1156-1166.

Gispert M, L Faucitano, MA Oliver, MD Guárdia, C Coll, K Siggens, K Harvey, A Diestre. 2000. A survey of preslaughter conditions, halothane gene frequency, and carcass and meat quality in five Spanish commercial abattoirs. Meat Sci 55, 97-106.

Guárdia MD, J Estany, S Balasch, MA Oliver, M Gispert, A Diestre. 2004. Risk assessment of PSE condition due to pre-slaughter conditions and RYR1 gene in pigs. Meat Sci 67, 471-478.

Guárdia MD, J Estany, S Balasch, MA Oliver, M Gisper, A Diestre. 2009. Risk assessment of skin damage due to preslaughter conditions and RYR1 gene in pigs. Meat Sci 81, 745-751.

ICA, Instituto Colombiano Agropecuario, Colombia. 2007. Resolución 2640 del 1 de octubre de 2007. Bogotá, Colombia.

MPS, Ministerio de la Protección Social ${ }^{a}$, Colombia. 2007. De- creto número 1500 de 4 de mayo de 2007. Bogotá, Colombia.

MPS, Ministerio de la Protección Social ${ }^{\mathrm{b}}$. Colombia. 2007. Resolución 4282 de 21 de noviembre de 2007. Bogotá, Colombia.

Mota-Rojas D, M Becerril, C Lemus, P Sánchez, M González, SA Olmos, R Ramírez, M Alonso-Spilsbury. 2006. Effects of mid-summer transport duration on pre- and post-slaughter performance and pork quality in Mexico. Meat Sci 73, 404-412.

Nanni Costa L, DP Lo Fiego, S Dall'Olio, R Davoli, V Russo. 2002. Combined effects of pre-slaughter treatments and lairage time on carcass and meat quality in pigs with different halothane genotypes. Meat Sci 61, 41-47.

OIE, Organización Mundial de la Salud Animal. 2012. Código Sanitario para los Animales Terrestres. Título 7.

Pérez MP, J Palacio, MP Santolaria, MC Aceña, G Chacón, M Gascón, JH Calvo, P Zaragoza, JA Beltrán, S GarcíaBelenguer. 2002. Effect of transport time on welfare and meat quality in pigs. Meat Sci 425-433.

Romero MH, JA Sánchez, C Gutiérrez. 2011ª. Evaluación de prácticas de bienestar animal durante el transporte de bovinos para sacrificio. Rev Salud Pública 13, 684-690.

Romero MH, MJR Paranhos da Costa, JA Sánchez. 2011 b. Bienestar animal: un compromiso de la cadena cárnica bovina. Biosalud 10, 71-86.

Romero MH, C Gutiérrez, JA Sánchez. 2012. Evaluación de contusiones como un indicador de bienestar animal durante el pre-sacrificio de bovinos. Rev Colomb Cienc Pecu 25, 267-275.

Rosenvold K, HJ Andersen. 2003. Factors of significance for pork quality: A review. Meat Sci 64, 219-237.

Strappini AC, JHM Metz, CB Gallo, B Kemp. 2009. Origin and assessment of bruises in beef cattle at slaughter. Animal 3, 728-736.

Strappini AC, K Frankena, JHM Metz, CB Gallo, B Kemp. 2012. Characteristics of bruises in carcasses of cows sourced from farms or from livestock markets. Animal 6, 502-509.

Turner SP, JM Farnworth, IMS White, S Brotherstone, M Mendl, P Knap, P Penny, AB Lawrence. 2006. The accumulation of skin lesions and their use as a predictor of individual aggressiveness in pigs. Appli Anim Behav Sci 96, 245-259.

Van de Perre V, L Permentier, S De Bie, G Verbeke, R Geers. 2010. Effect of unloading, lairage, pig handling, stunning and season on $\mathrm{pH}$ of pork. Meat Sci 86, 931-937.

Warriss PD. 2003. Optimal lairage times and conditions for slaughter pigs: a review. Vet Rec 153, 170-176.

Zhen S, Y Liu, X Li, K Ge, H Chen, C Li, F Ren. 2013. Effects of lairage time on welfare indicators, energy metabolism and meat quality of pigs in Beijing. Meat Sci 93, 287-291. 
